\title{
An investigation of the heart rate, heart rate variability, cardiac ions, troponin-I and CK-MB in men exposed to $1.5 \mathrm{~T}$ constant magnetic fields
}

\author{
Cemil Sert ${ }^{1}$, Zeynep Aktt ${ }^{2}$, Öcal Sirmatel ${ }^{3}$ and Remzi Yllmaz ${ }^{4}$ \\ ${ }^{1}$ Department of Biophysics, Faculty of Medicine, Harran University, Şanluurfa, Turkey \\ ${ }^{2}$ Department of Radiodiagnostics, Faculty of Medicine, Harran University, Şanluurfa, Turkey \\ ${ }^{3}$ Department of Radiodiagnostics, Faculty of Medicine, İzzet Baysal University, Bolu, Turkey \\ ${ }^{4}$ Department of Cardiology, Faculty of Medicine, Harran University, Şanluurfa, Turkey
}

\begin{abstract}
The aim of this study was to investigate heart rate (HR), heart rate variability (HRV) and other cardiac parameters in individuals who were exposed to a high static magnetic field. 30 healthy male volunteers aged between $20-30$ years were included.

The searching was divided into three phases: pre-magnetic field, during the magnetic field and post-magnetic field. Every phase lasted 30 minutes. Pulse, systolic and diastolic blood pressure, respiration rate and elektrocardiography (ECG), recorded for 30 minutes, in all of the individuals were measured during three phases. The men were exposed to a $1.5 \mathrm{~T}$ static magnetic field. The levels of $\mathrm{Na}^{+}, \mathrm{K}^{+}, \mathrm{Ca}^{2+}, \mathrm{Cl}^{-}, \mathrm{CK}-\mathrm{MB}$, troponin-I and $\mathrm{HR}$ and $\mathrm{HRV}$ parameters were investigated.

There was an increase in the respiration rate, and no change in the systolic and diastolic blood pressure and pulse in the individuals exposed to the magnetic field. There was also an enhancement in the values of ions, CK-MB and troponin-I after exposure to the magnetic field. Heart rate parameters (minimum HR-I, maximum HR-I, average HR-I) were decreased and rMSSD, pNN50, power, VLF, HF, LF values increased during the magnetic field.
\end{abstract}

Key words: Static magnetic field - Cardiac ions - Heart rate - Heart rate variability - Troponin-I $-\mathrm{CK}-\mathrm{MB}$

\begin{abstract}
Abbreviations: $\mathrm{HR}$, heart rate; $\mathrm{HRV}$, heart rate variability; $\mathrm{CK}-\mathrm{MB}$, myocardial creatine kinase; $\mathrm{NN}$, normal - normal interbeat interval; SDNN, standard deviation of NN intervals; SDANN, the standard deviation of the averaged NNs, calculated over short periods; rMSSD, the square root of the mean squared difference of successive NNs; NN50, the number of pairs of successive NNs that differ by more than $50 \mathrm{~ms}$; pNN50, the proportion of NN50 divided by total number of NNs; HF, high frequency; LF, low frequency.
\end{abstract}

\section{Introduction}

Magnetic resonance imaging (MRI) is one of the most common medical imaging methods in the world (Konez 1995). The common utilization of MRI in medicine necessitates the investigation of biological effects of high-level magnetic fields. The results of previous studies investigating these effects are varied. Generally it was reported that the magnetic field affected DNA, RNA and protein synthesis,

Correspondence to: Cemil Sert, Department of Biophysics, Faculty of Medicine, Harran University, Şanlıurfa, Turkey

E-mail: csert@harran.edu.tr cell division, $\mathrm{Ca}^{2+}$ influx across the plasma membrane, $\mathrm{Ca}^{2+}$ linking, signal transfer and other ion flows in the biological system (Cleary et al. 1993; Berg et al. 1999; Cridland et al. 1999). There are few studies on the effects of magnetic fields on ions and these studies are limited to $\mathrm{Ca}^{2+}$ and $\mathrm{K}^{+}$ (Sastre et al. 1998; Kangarlu et al. 1999; Ohata et al. 2004; Shen et al. 2007).

MRI utilizes a magnetic field, generally of a magnitude of 1.5 T. The strong static magnetic field used for MRI is thought to be detrimental to pacemaker function and it could potentially cause harm to patients undergoing MRI examinations. The potential adverse effects of MRI are: heating, rapid atrial pacing, rapid ventricular pacing, and asynchronous pacing. 
In some studies, the effects of a magnetic field on heart rate (HR) were investigated (Martin et al. 2004). However, the majority of these studies were carried out using $\mu \mathrm{T}$ and $\mathrm{mT}$ fields.

Savitz et al. (1999) detected occupational magnetic fieldrelated cardiovascular disease mortality in 140000 male electricity workers. They observed that in men who were exposed to magnetic fields for longer periods, mortality rate was higher as a result of arrhythmia and acute infarction. Cooper et al. (2009) did not provide evidence for an association between occupational magnetic field exposure and cardiovascular risc.

Chakeres and Vocht (2005) found important changes in the electrocardiogram (ECG) of subjects exposed to $1.5 \mathrm{~T}$ magnetic fields that increased with magnetic field strengths. The ECG returned to normal after the magnetic exposure.

In another study, in volunteers exposed to $200 \mathrm{mGauss}$ magnetic field were found significant changes in heart rate variability (HRV) (Sastre et al. 1998). Moreover, Tsuji et al. (1994) have observed decreases in various bands of HRV. HR was measured by detecting the intervals between the two QRS (electrical wave of cardiac ventricular muscle contraction). Sait et al. (2006) found that HR slowed down with exposure to magnetic field. They indicated that continuous exposure to a magnetic field affected both HR and HRV and, as the strength of the field increased, the effect became significant. Mitsutake et al. (2004) investigated whether 50 nT magnetic field could affect HR and HRV parameters. They observed that HR were reduced in subjects exposed to magnetic field without any important changes on the HRV parameters.

HRV can be defined as cyclic changes in sinus rate over time. It gives information about the sympatic and parasympatic balance; is an indicator of cardiac autonomic tonus and is used as an indicator of the cardio-respiratory system (Pieper et al. 1995; Malik 1998).

Previously, the cardiovascular performance of apes and dogs in a magnetic field were measured. No significant difference was observed in heart sound, heart valve movements, blood flow rate and intra-arterial blood pressure (Tenforde 1989).

Kangarlu et al. (1999) did not observe cardiogenic effects in pigs exposed to an $8 \mathrm{~T}$ magnetic field and any change in HRV in volunteers who were kept in an $8 \mathrm{~T}$ field for one hour. Otsuko et al. (2000) found a decrease in HRV in people who were exposed to a strong magnetic field.

The contraction and relaxation of the cardiac muscle occurs with movement of $\mathrm{Na}^{+}, \mathrm{K}^{+}, \mathrm{Ca}^{2+}$ and $\mathrm{Cl}^{-}$ions entering and exiting the cell membrane. Ion channels that serve in the movement of these ions are voltage-sensitive and may be affected by high static magnetic field. This may affect heart contraction and HR. However, there are few studies on ion movements within a high static magnetic field. Ion studies are limited to $\mathrm{Ca}^{2+}$ and $\mathrm{K}^{+}$. Examples include studies by Bawin et al. (1978), Blackman et al. (1985) and Bellosi et al. (1986). These three researchers investigated $\mathrm{Ca}^{2+}$ ion transfer in different tissues in a low-level magnetic field. There is not sufficient investigation concerning ion influx in cardiac ventricle cells in high static magnetic field.

The literature lacks a study which investigates HR, HRV, CK-MB (myocardial creatine kinase), troponin-I and $\mathrm{Na}^{+}, \mathrm{K}^{+}, \mathrm{Ca}^{2+}, \mathrm{Cl}^{-}$ions together. In addition, the time and frequency values of HRV have not been individually investigated.

The aim of this study was to investigate HR, HRV, cardiac ions, CK-MB and troponin-I in the individuals who were exposed to a short-term static magnetic field $(1.5 \mathrm{~T})$. For this reason, in the present study, we investigated all of these parameters in male volunteers exposed to a $1.5 \mathrm{~T}$ level of static magnetic field for 30 minutes.

\section{Materials and Methods}

\section{Study protocol}

Thirty non-smoking, healthy male volunteers aged 20-30 years participated in this study. The weight range of volunteers was $69.7 \pm 10.6 \mathrm{~kg}$ and subject's height was $172 \pm 5 \mathrm{~cm}$. Volunteers were chosen from healthy individuals according to our criteria. These individuals were studying in health high school (college). Their cardiovascular health was assessed based on an examination by a cardiologist. Participants did not have cardiological, neurological or vascular diseases, diabetes, hypertension, epilepsy, HR disorders, heart stent or cardiac pacemaker. Because of the hormonal and systemic changes caused by the menstrual cycle, females were not included in the study. The magnetic exposure was conducted between 8 a.m. and 12 a.m and subjects were fasting. The study was categorized into three groups: pre-exposure, during-exposure and post-exposure.

\section{Methods}

Before exposure, after 20 minutes of resting, pulse, systolic and diastolic blood pressure and respiration rates were measured for all volunteers. These measurements were repeated after exposure. Blood pressure was measured manually by cardiologist and was interpreted by same doctor.

During exposure, the volunteers were kept in the MRI environment for 30 minutes in a supine position without moving. During this period, MRI device was turned off to exclude the radio frequency (RF) pulse and pulsed magnetic gradient field in environment. The volunteers were only exposed to a $1.5 \mathrm{~T}$ static magnetic field (General Electric, Signa Excite, Milwaukee, USA). 
In the pre-exposure and post-exposure periods, $5 \mathrm{ml}$ blood was taken from an antecubital vein in sterile conditions. The blood samples were centrifuged for $10 \mathrm{~min}$ at a rate of 5000 revolutions/min (Heraeus, Megafuje 1.0, D-37520, Osterode, Germany), and plasma was seperated. $\mathrm{Na}^{+}, \mathrm{K}^{+}, \mathrm{Ca}^{2+}, \mathrm{Cl}^{-}$, troponin-I and CK-MB levels were measured from the separated serums. The ions were detected using an Abbot Aeroset autoanalyser (LN090D0501, Toshigi-Ken, Japan) and CK-MB and troponin-I was detected using BIO-PAC Immulite 2500 hormone device (Los Angeles, CA, USA). $\mathrm{Na}^{+}, \mathrm{K}^{+}, \mathrm{Cl}^{-}$ion levels were determined by ion selective electrode method, calcium ion level by spectrophotometric method, troponin-I and CK-MB levels by a solid-phase, enzyme-labeled chemiluminescent immunometric assay.

In the pre-exposure period, post-exposure period and during the exposure, a digital ECG record was made using DMS 300-7 (Nevada, USA) holter device for 30-minute periods. Holter records were analyzed using the holter software. HR (minimum HR-I, maximum HR-I, average HR-I) and HRV (SDNN, SDANN, rMSSD, pNN50, power, VLF, LF and HF) values were assessed by the same cardiologist. The time domain of $\mathrm{HRV}$ is SDNN, SDANN, rMSSD and pNN50. LF, HF and VLF are frequency domain of HRV.

Systolic and diastolic blood pressure, respiration frequency, pulse, $\mathrm{Na}^{+}, \mathrm{K}^{+}, \mathrm{Ca}^{2+}, \mathrm{Cl}^{-} \mathrm{CK}-\mathrm{MB}$, troponin-I parameter values of the volunteers before and after the application were compared. Respiration frequency of each volunteer was determined by inspection for one minute. Pre-exposure, during exposure and post-exposure values of HR and HRV (minimum HR-I, maximum HR-I, average HR-I, SDNN, SDANN, rMSSD, pNN50, power, VLF, LF and HF) parameters were compared to each other.

\section{Statistical Methods}

Statistical analyses were conducted using the SPSS 12.0 statistical software program. Paired $t$-test was used to analyze the difference between the mean values of $\mathrm{Na}^{+}, \mathrm{K}^{+}, \mathrm{Ca}^{2+}, \mathrm{Cl}^{-}$, $\mathrm{CK}-\mathrm{MB}$, troponin-I levels of two measures (pre-magnetic field and post-magnetic field) of the same individuals; and the general linear model repeated was used for the analysis of the difference between the mean values of HRV parameters of the three measures (pre-magnetic field, during magnetic field and post-magnetic field) of the same individuals. Tukey test was used for post hoc multiple comparisons of observed means.

The results were calculated as average \pm standard deviation. The significance level was set as $p<0.05$.

This study was approved by Harran University Ethics Committee in their letter dated 08/11/2006 and numbered 101.5/68.

\section{Results}

According to the pre-exposure and post-exposure analysis results, there was an insignificant decrease in systolic and diastolic blood pressure $(p>0.05)$, a statistical decrease in respiration number $(p<0.05)$ and a decrease in pulse, but in border $(p=0.05)$ (Table 1).

In the post-exposure period, a significant increase was observed in $\mathrm{Na}^{+}, \mathrm{K}^{+}, \mathrm{Ca}^{2+}, \mathrm{Cl}^{-}$and troponin-I values when compared to pre-exposure values $(p<0.01, p<0.05)$. The change in CK-MB was at the significance limit $(p=0.049)$ (Table 2).

HR, minimum HR-I, maximum HR-I and average HR-I values of the individuals significantly decreased during exposure when compared to the pre-exposure period $(p<0.05)$. No significant post-exposure increase was found when compared to measurements recorded during exposure $(p<0.05)$ and was not found any change between preexposure and post-exposure values $(p>0.05)$. In SDNN, SDANN, rMSSD, pNN50, power, VLF, LF, HF parameters of

Table 1. Comparison of heart parameters before and after the application of magnetic field

\begin{tabular}{lccc}
\hline Parameters & $\begin{array}{c}\text { Pre-magnetic } \\
\text { field }\end{array}$ & $\begin{array}{c}\text { Post-magnetic } \\
\text { field }\end{array}$ & $p$ \\
\hline $\begin{array}{l}\text { Systolic blood } \\
\text { pressure (mmHg) }\end{array}$ & $116 \pm 21$ & $112 \pm 22$ & 0.206 \\
$\begin{array}{l}\text { Diastolic blood } \\
\text { pressure (mmHg) }\end{array}$ & $67 \pm 12$ & $65 \pm 12$ & 0.480 \\
$\begin{array}{l}\text { Respiration } \\
\text { (number/min) }\end{array}$ & $18.8 \pm 2.8$ & $20.1 \pm 3.3$ & 0.011 \\
$\begin{array}{l}\text { Pulse } \\
\text { (number/min) }\end{array}$ & $80 \pm 11$ & $76 \pm 10$ & 0.050 \\
\hline
\end{tabular}

Values \pm S.D., $n=30,{ }^{\mathrm{a}} p>0.05 v s$. pre-magnetic field; ${ }^{\mathrm{b}} p<0.05$ $v s$. pre-magnetic field

Table 2. Values of cardiac ions and CK-MB and troponin-I in premagnetic field and post-magnetic field

\begin{tabular}{|c|c|c|c|}
\hline Parameters & $\begin{array}{l}\text { Pre-magnetic } \\
\text { field }\end{array}$ & $\begin{array}{l}\text { Post-magnetic } \\
\text { field }\end{array}$ & $p$ \\
\hline $\mathrm{Na}^{+}(\mathrm{mEq} / \mathrm{l})$ & $140 \pm 3.7$ & $145 \pm 2.38$ & 0.001 \\
\hline $\mathrm{K}^{+}(\mathrm{mEq} / \mathrm{l})$ & $4.0 \pm 0.4$ & $4.2 \pm 0.4$ & 0.026 \\
\hline $\mathrm{Cl}^{-}(\mathrm{mEq} / \mathrm{l})$ & $109 \pm 2.5$ & $114 \pm 1.70$ & 0.001 \\
\hline $\mathrm{Ca}^{2+}(\mathrm{mEq} / \mathrm{l})$ & $9.3 \pm 0.4$ & $9.9 \pm 0.45$ & 0.001 \\
\hline CK-MB (U/l) & $2.7 \pm 1.8$ & $3.1 \pm 1.9$ & 0.049 \\
\hline troponin-I $(\mu \mathrm{g} / \mathrm{l})$ & $0.025 \pm 0.006$ & $0.03 \pm 0.01$ & 0.012 \\
\hline
\end{tabular}

It was found that average $\mathrm{Na}^{+}, \mathrm{K}^{+}, \mathrm{Cl}^{-}, \mathrm{Ca}^{2+}, \mathrm{CK}-\mathrm{MB}$ and troponin-I values increased significantly after the application $(p<0.05)$. $p$ value of average CK-MB changed at the level of the significance threshold. Values \pm S.D., $n=30$. 
HRV were observed an increase during the exposure when compared to pre-exposure $(p<0.05)$, and these parameters decreased post exposure compared to during exposure $(p<$ $0.05)$. No significant difference was determined in any of the parameters between the mean values of post-exposure and pre-exposure $(p<0.05)$ (Table 3$)$.

According to these findings, it was observed that HR parameters decreased with exposure; they returned to normal within the post-exposure period; time and frequency-based HRV parameters increased with exposure and returned to normal within the post-exposure period.

\section{Discussion}

A high static magnetic field may affect the ions in the body. This may cause biopotential formation in muscle and nerve cells (Formica and Silvestri 2004).

The intervals $(\mathrm{N}-\mathrm{N})$ between two successive normal beats from the sinoatrial node are evaluated as time-based parameters. These are SDNN, SDANN, rMSSD, pNN50 indexes. Frequency-based parameters give information about all variability amounts in HR. These are LF, HF, VLF bands (Malpas et al. 1990; Binkley et al. 1993; Karaso et al. 1999; May et al. 2000).

Table 3. Parameters of HR and time-based and frequency-based parameters of HRV during pre-magnetic field, magnetic field and post-magnetic field

\begin{tabular}{lccc}
\hline Parameters & $\begin{array}{c}\text { Pre-mag- } \\
\text { netic field }\end{array}$ & $\begin{array}{c}\text { During } \\
\text { magnetic } \\
\text { field }\end{array}$ & $\begin{array}{c}\text { Post-magnetic } \\
\text { field }\end{array}$ \\
\hline min HR-I (bpm) & $62.6 \pm 7.1$ & $54.3 \pm 7.2$ & $62.3 \pm 8.9$ \\
max HR-I (bpm) & $98.8 \pm 14.7$ & $87.2 \pm 23.0$ & $94.8 \pm 17.3$ \\
average HR-I (bpm) & $77.1 \pm 8.4$ & $65.9 \pm 9.1$ & $72.8 \pm 15.9$ \\
SDNN (msn) & $70.4 \pm 12.1$ & $84.6 \pm 15.0$ & $67.0 \pm 16.2$ \\
SDANN (msn) & $27.4 \pm 5.1$ & $51.4 \pm 6.0$ & $22.6 \pm 5,8$ \\
rMSSD (msn) & $32.4 \pm 8.4$ & $46.3 \pm 9.94$ & $33.6 \pm 8.1$ \\
pNN50 (\%) & $11.8 \pm 3.2$ & $23.5 \pm 7.8$ & $13.0 \pm 4.7$ \\
power & $1956 \pm 322$ & $3155 \pm 396$ & $1454 \pm 04$ \\
VLF (msn) & $1139 \pm 232$ & $1858 \pm 336$ & $684 \pm 50$ \\
LF (msn) & $641 \pm 37$ & $882 \pm 79$ & $501 \pm 22$ \\
HF (msn) & $152 \pm 60$ & $371 \pm 77$ & $122 \pm 39$ \\
\hline
\end{tabular}

Heart rate values \pm S.D. and average heart rate variability values \pm S.D. of the volunteers, $n=30$; SDNN, standard deviation of all R-R intervals (top point of nearest two QRS in ECG); SDANN, standard deviation of average N-N intervals; $\mathrm{rMSSD}$, average square root of consecutive differences; pNN50, total number of all R-R intervals; $\mathrm{HF}$, high frequency heart rate; $\mathrm{LF}$, low frequency heart rate; $\mathrm{NN}$, normal to normal interbeat interval; $p<0.05$, pre-exposure period $v s$. during exposure; $p<0.05$, during exposure $v s$. post-exposure period; $p>0.05$, pre-exposure period $v s$. post-exposure period.
Sastre et al. (1998) and Mardi et al. (1999) observed a decrease in the LF band of HRV, and an increase in HF band of HRV in people who were exposed to a sinusoidal magnetic field. The decrease in LF and the increase in HF are clinically related with cardiovascular diseases and can be life threatening. HF is only related to parasympathetic effects; while LF is related with both sympathetic and parasympathetic effects. The decrease in LF may be related to increased vagal and acetylcholine activation (Winchel et al. 1996; Cook et al. 1997). In some large prospective cohort studies it is assumed that, as a result of the decreases in specific components of HRV, a heart disease develops (Dekker et al. 1997; Cook et al. 1997; Martin et al. 2004). The decreased HRV amongst people who had a past myocardial infarct is related to the increased risk of all causes of death (Lombardi et al. 1987; Ori et al. 1992; Vaishnav et al. 1994; Bigger et al. 1995).

In another study, Sastre et al. (1998) observed a decrease in the LF band and an increase in the HF band in a 200 mGauss magnetic field. However, when the magnetic field was applied at intervals rather than continuously, they observed no change in either band. Some researchers reported a decrease in HF band and a minor change or no change in others (Winchell et al. 1996; Barron et al. 1996; Huikuri et al. 1996). These studies were carried out in low magnetic fields. There are few studies on this subject which have used a Tesla-level static magnetic field.

In many studies, reduced LF and increased HF were observed. In our study, with exposure to high static magnetic field was observed increase in LF and HF. It is possible that a high static magnetic field might affect both the symphatic and parasymphatic systems. The observed reduction in HR with exposure to high magnetic field can be associated with increased vagal and therefore acetylcholine activation. Alternatively receptor numbers or sensitivities might be altered. Exposing to a high static magnetic field an increased motion of ions and voltage-induced ion channels can occure. Serum potassium and troponin-I may be an indicator of myocardial damage (Kangarlu et al. 1999). In our study, we observed an increase in serum potassium and troponin-I. This may also be a risk to cardiac system. They did not observe significant changes in body temperature, HR, systolic pressure, diastolic pressure, troponin-I and potassium levels following 3 hours of exposure to a field strength of 8.0 Tesla (Kangarlu et al. 1999). Their systolic and diastolic pressure values are in accordance with our results. HR, potassium and troponin results are not in agreement with ours results. They measured all parameters after 3 hours of exposure to the magnetic field. If they had measured immediately after exposure to the magnetic field, their results might have differed.

In the present study, the respiratory rate increased significantly. The increased respiration rate is consistent with the significant increase in the HF band of HRV, because the change in the HF band is related to parasympathetic activity 
and is affected by respiration. In some studies mentioned above, a decrease in the LF band and an increase in the HF band were reported. The changes in LF and HF are clinically related with cardiovascular diseases. The LF band is related to blood pressure and thermoregulator control. The change in the LF band is the precursor of cardiovascular morbidity and mortality (Tsuji et al. 1996).

Ohata et al. (2004) observed that exposure to $0.4 \mathrm{~T}$ magnetic field significantly increased $\mathrm{K}^{+}$flow from cell membrane. We investigated also $\mathrm{Na}^{+}$and $\mathrm{Cl}^{-}$levels in addition to $\mathrm{Ca}^{2+}$ and $\mathrm{K}^{+}$. In our study, all of these ions significantly changed after the exposure. These two studies were in accord with each other. External electric and magnetic fields create a force on the ions inside channel protein and the free ions on the two sides of the plasma membrane. This external force may change the direction of random movement (Panagopoulos et al. 2002). This kind of change in ion movements can trigger HR and HRV change.

According to the results of the present study, in male volunteers who were exposed to $1.5 \mathrm{~T}$ static magnetic fields, respiration rate significantly increased and pulse exhibited an increase which was at the significance threshold. Values of $\mathrm{Na}^{+}, \mathrm{K}^{+}, \mathrm{Ca}^{2+}, \mathrm{Cl}^{-}, \mathrm{CK}-\mathrm{MB}$, troponin-I all increased significantly after the exposure. HR parameters were decreased with exposure; HRV parameters increased with the exposure; however, both HR and HRV returned to normal after the exposure. The increase of HRV can be indicative of a degeneration of sympatovagal balance and may be an indicator of cardiac arrhythms. The increase in CK-MB and troponin-I levels may be caused by reversible or irreversible myocardium damage (Murray et al. 2004). An increase in CK-MB and troponin-I levels was also observed in our study. These results indicate that exposures of 30 minutes affect HR and HRV. This implies that the cardiovascular system response is immediate and acute exposure to $1.5 \mathrm{~T}$ static magnetic field can also be dangerous for the cardiac system.

This study is a pre-post investigation in same subject. Subjects were students of high health school and they are experienced in medical process. Moreover, they had closed their eyes during magnetic field exposure. Therefore, we think that on the subjects can't be emotional effect of magnetic field. Because, the control group would be remain in the MRI. Sastre et al. (1998), forty healthy young men included to investigation. In their study, each subject served as his own control. Also in our study, each subject was his own control.

These parameters were not previously investigated together. For this reason, the results of this study give important information about interaction of cardiac system with high static magnetic field. However, for full explanation of this subject, it is necessary to determine acetylcholine, epinephrine, norepinephrine levels and heart muscle intra- cellular $\mathrm{Na}^{+}, \mathrm{K}^{+}, \mathrm{Ca}^{2+}, \mathrm{Cl}^{-}$levels with the above mentioned parameters. Recording HR and HRV using holter is quite difficult in animals. Therefore, these parameters should be carried out separately on animals and on humans and the results should be interpreted together.

Acknowledgement. This study was supported by the Scientific Research Fund of Harran University (Project No. 702).

\section{References}

Bawin S. M., Adey W. R., Sabbot I. M. (1978): Ionic factors in release of $45 \mathrm{Ca} 2+$ from chicken cerebral tissue by electromagnetic fields. Proc. Natl. Acad. Sci. U.S.A. 75, 6314-6318; doi:10.1073/pnas.75.12.6314

Barron H. U., Lesh M. D. (1996): Autonomic nervous system and sudden cardiac deat. J. Am. Coll. Cardiol. 27, 1053-1060; doi:10.1016/0735-1097(95)00615-X

Berg H. (1999): Problems of weak electromagnetic field effect in cell biology. Bioelectrochem. Bioenerg. 48, 355-360; doi:10.1016/S0302-4598(99)00012-4

Bellossi A. (1986): Lack of an effect of static magnetic field on calcium efflux from isolated chick brain. Bioelectromagnetics 7, 381-386; doi:10.1002/bem.2250070405

Binkley P. F., Haas G. J., Starling R. C. (1993): Sustained augmentation of parasympathetic tone with angiotensin converting enzyme inhibition in patients with congestive heart failure. J. Am. Coll. Cardiol. 21, 655-661; doi:10.1016/07351097(93)90098-L

Blackman C. F., Benane S. G., House D. E., Joines W. T. (1985): Effects of ELF $(1-120 \mathrm{~Hz})$ and modulated $(50 \mathrm{~Hz}) \mathrm{RF}$ fields on the efflux of calcium ions from brain tissue in vitro. Bioelectromagnetics. 6, 1-11; doi:10.1002/ bem. 2250060102

Chakeres D. W., Vocht F. (2005): Static magnetic field effects on human subjects related to magnetic resonance imaging systems. Prog. Biophys. Mol. Biol. 87, 255-265; doi:10.1016/j.pbiomolbio.2004.08.012

Cleary S. F. (1993): A review of in vitro studies: low-frequency electromagnetic fields. Am. Ind. Hyg. Assoc. J. 54, 178-185; doi:10.1080/15298669391354531

Cook M. R., Graham C., Sastre A., Hoffman S. J., Gerkovich M. M. (1996): Gender differences in heart rate variability in 60 Hz magnetic fields. J. Physchophysiol. 33, S30

Cooper A. R., Van Winjgaarden E., Fisher S. G., Adams J. M., Yost M. G., Bowman J. D. (2009): A population-based cohort Study of Occupational Exposure to Magnetic Fields and Cardiovascular Disease Mortality. Ann. Epidemiol. 19, 42-48; doi:10.1016/j.annepidem.2008.10.001

Cridland N. A., Sabour N. R., Saunders R. D. (1999): Effect of 50 $\mathrm{Hz}$ magnetic field exposure on the rate of RNA synthesis by normal fibroblast. Int. J. Radiat. Biol. 75, 647-654; doi:10.1080/095530099140294

Dekker J. M., Schouten E. G., Klootwijk P., Pool J., Swenne C.A., Kromhout D. (1997): Heart rate variability from short electrocardiographic ecordings predicts mortality from 
all causes in middle-aged and elderly men. Am. J. Epidemiol. 145, 899-908

Formica D., Silvestri S. (2004): Biological effects of exposure to magnetic resonance imaging: an overview. Biomed. Eng. Online 3, 1-20; doi:10.1186/1475-925X-3-11

Huikuri H. V., Ylitalo A., Pikkujamsa S. M., Ikaheimo M. J., Airaksinen K. E., Rantala A.O., lilja M., Kesaniemi Y. A. (1996): Heart rate variability in systemic hypertension. Am. J. Cardiol. 77, 1073-1077; doi:10.1016/S0002-9149(96)00135-X

Kangarlu A., Burgess R. E., Zhu H., Nakayama T., Hamlin R. L., Abduljalil A. M., Robitaille P. M. (1999): Cognitive, cardiac and physiological safety studies in ultra high field magnetic resonance imaging. Magn. Reson. Imaging 17, 1407-1416; doi:10.1016/S0730-725X(99)00086-7

Karason K., Molgaard H., Wikstrand J., Sjostrom L. (1999): Heart rate variability in obesity and the effect on weight loss. Am. J. Cardiol. 83, 1242-1247; doi:10.1016/S00029149(99)00066-1

Konez O. (1995): Magnetic Resonance Imaging. Nobel medical bookstore, Istanbul

Lombardi F., Sandrone G., Pernpruner S., Sala R., Garimoldi M., Cerutti S., Baselli G., Pagani M., Malliani A. (1987): Heart rate variability as an index of sympathovagal interaction after acute myocardial infarction. Am. J. Cardiol. 60, 1239-1245; doi:10.1016/0002-9149(87)90601-1

Malik M. (1998): Heart rate variability. Curr. Opin. Cardiol. 13, 36-44; doi:10.1097/00001573-199801000-00006

Malpas S. C., Maling T. J. (1990): Heart rate variability and cardiac autonomic function in diabetes. Diabetes 39, 1177-1181; doi:10.2337/diabetes.39.10.1177

Martin E. T., Coman J. A., Shellock F. G., Pulling C. C., Fair R., Jenkins K. (2004): Magnetic resonance imaging and Cardiac pacemaker safety at 1.5 Tesla. J. Am. Coll. Cardiolog. 43, 1315-1324; doi:10.1016/j.jacc.2003.12.016

May O., Arildsen H. (2000): Assessing cardiovascular autonomic neuropaty in diabetes mellitus: how many tests to use? J. Diabetes Complicat. 14, 7-12; doi:10.1016/S10568727(00)00062-3

Mitsutake G., Otsuka K., Oinuma S., Ferguson I., Cornelissen G., Wanliss J., Haldberg F. (2004): Does exposure to an artificial ULF magnetic field affect blood pressure, heart rate variabilityand mood? Biomed. Pharmacother. 58, S20-27; doi:10.1016/S0753-3322(04)80004-0

Murray R. K., Granner D. K., Mayes P. A., Rodwell V. W. (2004): Harper Biyokimya. Nobel Medical Bookshop, Ankara

Ohata R., Tomita N., Ikada Y. (2004): Effect of a static magnetic field on ion transport in a cellulose membrane. J. Colloid Interface Sci. 270, 413-416; doi:10.1016/j.jcis.2003.09.035

Ori Z., Monir G., Weiss J. (1992): Heart rate variability. Frequency domain analysis. Cardiol. Clin. 10, 499-537

Otsuka K., Yamanaka T., Cornellissen G., Breus T., Chibisov S. M., Baevsky R., Siegelova J., Fiser B., Halberg F. (2000):
Altered chronoma of heart rate variability during span of high magnetic activity. Scripta Medica 73, 111-116

Panagopoulos D. J., Karabarbounis A., Margaritis L. H. (2000): Mechanism for action of electromagnetic fields on cells. Biochem. Biophys. Res. Commun. 298, 95-102; doi:10.1016/S0006-291X(02)02393-8

Pieper S. J., Hamil S. C. (1995): Heart rate variability: tecnique and investigational applications in cardiovascular medicine. Mayo Clin. Proc. 70, 955-964; doi:10.4065/70.10.955

Sait M. L., Wood A.W., Kirscner R. L. (2006): Effects of $50 \mathrm{~Hz}$ magnetic field exposure on human heart rate variability with passive tilting. Physiol. Meas. 27, 73-83; doi:10.1088/ 0967-3334/27/1/007

Sait M. L., Wood A. W., Sadafi H. A. (1999): A study of heart rate and heart rate variability in human subjects exposed to occupational levels of $50 \mathrm{~Hz}$ circularly polarized magnetic fields. Med. Eng. Phys. 21, 361-369; doi:10.1016/S13504533(99)00062-4

Sastre A., Cook M. R., Graham C. (1998): Nocturnal exposure to intermittent $60 \mathrm{~Hz}$ magnetic fields alters human cardiac rhythm. Bioelectromagnetics 19, 98-106; doi:10.1002/(SICI)1521-186X(1998) 19:2<98::AID-BEM7>3.0.CO;2-Z

Savitz D. A., Liao D., Sastre A., Kleckner R. C., Kavet R. (1999): Magnetic field exposure and cardiovascular disease mortality among electric utility workers. Am. J. Epidemiol. 149, 135-142

Shen J. F., Chao Y. L., Du L. (2007): Effects of static magnetic fields on the voltage gated potassium channel currents in trigeminal root ganglion neurons. Neurosci. Lett. 415, 164-168; doi:10.1016/j.neulet.2007.01.015

Tenforde T. S. (1989): Biological responses to static and time-varying magnetic fields. In: Electromagnetic Interaction with Biological Systems. (Ed. J. C. Lin), pp. 83-107, Plenum, New York

Tsuji H., Venditti F. J., Manders E. S., Evans J. C., Larson M. G., Feldman C. L., Levy D. (1994): Reduced heart rate variability and mortality risk in an elderly cohort. The Framingham Heart Study. Circulation 90, 878-883

Tsuji H., Larson M. G., Venditti F. J., Manders E. S., Evans J. C., Feldman C. L., Levy D. (1996): Impact of reduced heart rate variability on risk for cardiac events. The Framingham Heart Study. Circulation 94, 2850-2855

Vaishnav S., Stevenson R., Machnat B. (1994): Relation between heart rate variability early after acute myocardial infarction and long-term mortality. Am. J. Cardiol. 73, 653-657; doi:10.1016/0002-9149(94)90928-8

Winchell R. J., Hoyt D. B. (1996): Spectral analysis of heart rate variability in the ICU: a measure of autonomic function. J. Surg. Res. 63, 11-16; doi:10.1006/jsre.1996.0214

Received: February 26, 2010

Final version accepted: May 5, 2010 